Results A 27-year-old healthy women with an history of cervical cerclage at 26 weeks, required a new cervical cerclage at 29 weeks. Spinal anesthesias were performed. In the first surgery a $27 \mathrm{G}$ pencil point was used without complications. In the second surgery, 3 attempts were required with a $27 \mathrm{G}$ quincke, but as the block failed, general anesthesia was necessary.

Two days after surgery, she developed frontal headache, vomiting, photophobia and noise sensibility that aggravated by orthostatic position. She was apyretic, normotensive and with no neurological deficits. The blood tests were normal except for anemia. Conservative treatment for PDPH was tried with no success and she was offered an epidural blood patch (EBP). The procedure, the risks and benefits were explained. Under aseptic condition the EBP was performed with $20 \mathrm{ml}$ of autologous blood with no complications. The procedure was successful and she was discharged home asymptomatic.

One month later she was admitted with full dilatation. Labor analgesia with epidural was effective.The postoperative course was uneventful.

Conclusions In our case, multiple attempts and the use of a quincke needle increased the risk of PDPH. Even though the time between EBP and the subsequent epidural analgesia was only one month, we speculate that the EBP had no effect on the quality of epidural analgesia.

\section{VAGINAL BIRTH AFTER CESAREAN SECTION IN ALBANIA}

${ }^{1} \mathrm{~N}$ Ceni", ${ }^{1} \mathrm{~A}$ Bimbashi, ${ }^{2} \mathrm{R}$ Ceni. 'Obstetric-Gynecological University Hospital 'Koço Gliozheni', Tirana, Albania; ${ }^{2}$ Lady of Good Counsel University, Tirana, Albania

\subsection{6/rapm-2021-ESRA.123}

Background and Aims The rate of primary cesarean section (CS) is on the rise. More and more women report with a history of a previous CS. A trial of vaginal delivery can save these women from the risk of repeat CS. The study was conducted to assess the safety and success rate of vaginal birth after CS (VBAC) in selected cases of one previous lower segment CS (LSCS).

Methods This is a prospective study conducted at the Department of Obstetrics and Gynecology, University Hospital 'Koço Gliozheni', Tirana, Albania, in the period 2015-2019. Sixty eight pregnant women with a history of one previous LSCS were enrolled in the study. The study was approved by national Medical Ethics Committee of Albania.

Results In the present study, 92\% cases had a successful VBAC and $8 \%$ underwent a repeat emergency LSCS for failed trial of vaginal delivery. Cervical dilatation of more than $3 \mathrm{~cm}$ at the time of admission was a significant factor in favor of a successful VBAC. Birth weight of more than 3,000g was significantly associated with a lower success rate of VBAC $(p<0.01)$. The incidence of fatal distress and uterine rupture was $1.6 \%$ respectively in the present study. There was no maternal or neonatal mortality.

Conclusions Trial of VBAC in selected cases has a great importance in the present era of the rising rate of primary CS. Majority of the cases of previous CS done for nonrecurrent indication can be delivered safely by the vaginal route, without any major complication to the mother and the newborn.

\section{4 ANAESTHETIC APPROACH IN PREGNANTS INFECTED WITH COVID-19 IN A TERTIARY MATERNITY}

AF Correia*, S Ramos, I Vilhena, I Martins, S Sá, L Melo, I Silva. CHUC, Coimbra, Portugal

\subsection{6/rapm-2021-ESRA. 124}

Background and Aims The authors analyse the anaesthetic approach in pregnants infected (PI) with COVID-19 admitted in a tertiary maternity (TM) during 12 months. They report and compare data from three-month period and data from not infected pregnants (NIP).

Methods Descriptive and retrospective observational study, with no need to ethical approval according to local committee. Data from a sample of 2288 pregnants (April 1,2020 March 31,2021). Variables and their associations were analysed with statistical tests using the SPSS tool.

Results $2.8 \%$ of pregnants were infected with COVID-19. $11 \%$ of these were admitted in the $1^{\text {st }}$ quarter, $2 \%$ in $2^{\text {nd }}$, $29 \%$ in $3^{\text {rd }}$ and $58 \%$ in $4^{\text {th }}$.

The anaesthetic approach was, with statistically significant, different between PI and PNI $(\mathrm{p}<, 001)$. Use of neuraxial labour analgesia/anaesthesia in PI increased $\left(42,9 \%-1^{\text {st }}\right.$ vs $\left.65,8 \%-4^{\text {th }}\right)$. General anaesthesia decreased $\left(10,5 \%-1^{\text {st }}\right.$ vs $\left.5,3 \%-4^{\text {th }}\right)$. Comparing deliveries, there was a statistically significant difference $(\mathrm{p}=0.01): 38 \%$ of births were caesarean section (CS) in PI vs $23 \%$ in PNI. In PI there was a decrease of $8,7 \%$ in CS between the $1^{\text {st }}$ and $4^{\text {th }}$ quarter. The need for emergency surgery (ES) had statistically significant difference $(p=0.02), 26.2 \%$ of ES in PI vs $15.1 \%$ in PNI. In PI there was no statistically significant difference $(p>0,05)$ when comparing the occurrence of ES in the different quarters.

Conclusions An increase of neuraxial labour analgesia/anaesthesia and decrease in GA was verified, despite the higher percentage of ES in PI. This can be related to increase in experience and knowledge, demostrating that neuraxial thecniques are safe/recommended in pregnants infected with COVID-19.

\section{CONTENTS OF URIC ACID, VITAMIN D IN THE BLOOD SERUM AND CHARACTERISTICS OF EPIDURAL ANALGESIA FOR LABOR IN PARTURIENT WOMEN WITH PREECLAMPSIA}

E Oreshnikov*, S Oreshnikova, E Vasiljeva, T Denisova. Chuvash State University, Cheboksary, Russian Federation

\subsection{6/rapm-2021-ESRA. 125}

Background and Aims It is known that more than $40 \%$ of pregnant women have a deficiencyof vitamin D. In addition to the classic symptoms of preeclampsia, more than a quarter of century, many foreign clinicians as preeclampsia indicator used by the hyperuricemia. We study the relationship of pain in childbirth, characteristics of epidural analgesia in patients with preeclampsia and the level of vitamin $\mathrm{D}$, uric acid in the blood serum.

Methods The study group included patients with severe and moderate preeclampsia,alone have given birth vaginally with epidural analgesia. The control group included patients with physiological pregnancy, independently gave birth vaginally with epidural analgesia. The material of the study was to peripheral blood of pregnant women taken at admission to 